\title{
Alternativas para la Adquisición de Datos Colaborativos Necesarios en la Construcción de Mapas de Ruido de Trafico Automotor
}

\author{
Alternatives for the collaborative data acquisition required at the automotive traffic noise maps \\ Jonathan Ochoa Villegas ${ }^{1 *}$, Jhon Sergio Marulanda Orozco ${ }^{1}$ y Luis Alberto Tafur Jiménez ${ }^{1}$
}

\begin{abstract}
Resumen
La construcción de mapas de ruido por métodos de cálculo requiere el levantamiento de datos geográficos para conformar las bases datos geográficas, involucrando personal y equipo técnico especializado. Actualmente, existen iniciativas colaborativas de las tecnologías de la información y de la comunicación que permiten compartir y editar geodatos para potenciar proyectos ambientales de gestión de ruido. De igual forma, las ciudades integran sistemas de seguridad y movilidad con datos colaborativos, permitiendo caracterizar los geodatos asociados al modelado del ruido. Por ello, en este artículo se presentan una iniciativa para la recolección de geodatos y estrategias para la estimación de atributos asociados a fuentes de ruido para generar mapas de ruido. Además, se presenta un caso de estudio del proceso para validar las iniciativas y las estrategias propuestas.

Abstract

The construction of noise maps by calculation methods requires the lifting of geographical data to form the geographic data bases, involving personnel and specialized technical equipment. Currently, there are collaborative initiatives of information and communication technologies that allow sharing and editing geodata to promote environmental noise management projects. In the same way, the cities integrate security and mobility systems with collaborative data, allowing to characterize the geodata associated with noise modeling. Therefore, this article presents an initiative for the collection of geodata and strategies for estimating attributes associated with noise sources to generate noise maps. In addition, a case study of the process is presented to validate the proposed initiatives and strategies.

Palabras Clave

Ciudades Inteligentes; gestión del ruido; mapas colaborativos; mapas estratégicos de ruido.

Key words

Collaborative maps; noise management; smart cities, strategic noise.

${ }^{1}$ Facultad de ingenierías, Universidad de San Buenaventura, Medellín, Colombia

*Autor correspondiente: jonathan8avillegas@hotmail.com

Manuscrito recibido 10-02-2017; revisado 28-04-2017; aceptado 11-05-2017.
\end{abstract}

\section{Introducción}

En el Congreso Mundial del Medio Ambiente realizado en Estocolmo de 1972, organizado por la O.N.U., se declaró el ruido como agente físico y socioeconómico contaminante que influye en la calidad del medio [1]. La Organización Mundial de Salud (O.M.S) estudia el ruido ambiental y sus efectos con lo que establece guías para el ruido urbano relacionadas con la salud [2]. Estas son la base para definir normas para el manejo del ruido ambiental [3]. De igual forma, el Grupo de Trabajo de la Comisión Europea para la evaluación de la exposición al ruido (WG-AEN), publica en el año 2006 la segunda versión de las guías prácticas para la elaboración de mapas estratégicos del ruido y la producción de datos asociados a la exposición al ruido [4].

Los mapas de ruido, son la representación de una situación acústica existente o pronosticada en función de un indicador de ruido. Los mapas estratégicos de ruido realizan evaluaciones globales de la exposición al ruido producto de diferentes focos [5]. Estos tienen como finalidad ser la base para identificar los niveles de ruido ambiental a los cuales están expuestos la población [6]. Con ellos es posible definir y 
desarrollar planes, proyectos y programas para la prevención y seguimiento del ruido ambiental [7].

Desafortunadamente, existen muchos territorios consolidados, en los cuales es difícil y costoso, a partir del redesarrollo urbano, garantizar niveles de exposición al ruido adecuada. Sin embargo, una buena gestión de la información puede aportar a planes de gestión para disminuir la exposición al ruido y los efectos del mismo en la población.

A nivel urbano la evaluación del ruido ambiental se realiza por medio de mapas de ruido construidos a partir de mediciones o modelos de predicción sonora. Con ellos se pretende evaluar una zona para cuantificar la población expuesta a diferentes niveles de ruido. De esta manera, es una herramienta de gestión del ruido ambiental para la valoración de la exposición sonora de la población [8]. La inclusión de los ciudadanos en la construcción de los mapas de ruido permite una recolección ágil y menos costosa que típicas campañas de toma de datos de campo.

Para la construcción de mapas de ruido la información solicitada relevante gira en torno a la cartografía, datos de tráfico y de población. Diferentes modelos informáticos permiten realizar mapas de ruido de manera ágil, su calidad depende de la calidad y precisión de los datos suministrados al modelo [9]. Guías metodológicas como la publicada en mayo de 2005 por el Gobierno Español establecen de los datos de partida para la realización de mapas de ruido son: Cartografía digital, ortofotografías en color, modelos digitales de terreno, carreteras, ferrocarriles, focos industriales u otros focos puntuales, edificaciones, población y usos del suelo [10]. La obtención de este tipo de información generalmente resulta costosa para las entidades encargas de la construcción de mapas de ruido [11]. Actualmente, la evolución tecnológica permite que las personas tengan la posibilidad de informar y ser informados. En los últimos años, se populariza, con éxito, el uso de computadores, smartphones, tablets, asistentes digitales personales y portátiles permitiendo la recolección de datos en diversos campos [12]. El impacto de la tecnología en la sociedad permite que cada vez exista mayor conectividad, por lo que el uso de tecnologías libres y de código abierto se convierte en el enfoque con mayor potencial $[13,14]$.

Este artículo se centra en las herramientas tecnológicas tipo colaborativas que aportan a la recolección de datos para la construcción de mapas de ruido. Además, se describen tres iniciativas de código abierto (open source) para la obtención de datos geográficos, y atributos asociados con el modelamiento del ruido ambiental. También, se describen los mapas colaborativos construidos a partir de proyectos colaborativos como WAZE y Open Street Map. Así mismo, se abordan proyectos como SRTM (Shuttle Radar Topography Mission) de la NASA que permite obtener curvas de nivel de manera gratuita. Por último, la recolección de datos asociada con el tráfico rodado se aborda por la implementación de algoritmos de analítica de video para estimar aforos vehiculares y velocidad media vehicular.

\section{Mapas base colaborativos}

Con el lanzamiento de Google Maps en el año 2005, se propone un cambio considerable de la forma en que la humanidad manipula los mapas [15]. Hasta esa fecha se están realizando diferentes esfuerzos para lograr el uso masificado de mapas. Sin embargo, estos solo logran permear algunas organizaciones que con usuarios expertos y herramientas de software comercial logran el acceso a mapas digitales al interior de las organizaciones. Sin duda, existen otros esfuerzos y proyectos académicos y comerciales que aportan de manera significativa a la forma en que el mundo moderno se relaciona con los mapas. El acceso a este tipo de información, hoy en día, se potencia por dispositivos como Smartphones [16].

En el área de los SIG (Sistemas de Información Geográfica), diferentes empresas enmarcan la temática con grandes aportes. En primer lugar está Enviromental System Research Institute (ESRI) que desde el año 1970 incursiona en el mercado de los SIG con el Sofware ArcInfo, el cual evoluciona a lo que se conoce hoy en día como ArcGIS [17]. En segundo lugar se encuentra la empresa Intergraph con su producto Geomedia, que en el 2010 fue adquirida por Hexagon, seindo pionera en el manejo de herramientas de AM/FM Automated Mapping/Facilities Managment y que en conjunto con la empresa Bentley y su producto Microstation aportaron al desarrollo de la industria SIG [18]. En tercer lugar, está la empresa Autodesk con sus grandes aportes en SIG con los productos Autocad Map.

Por otro lado, las iniciativas de código abierto logran avances importantes en el campo de los SIG. Un ejemplo claro se encuentra en las herramientas QGIS y POSTGIS, las cuales liberan el uso y manipulación de datos espaciales de manera económica que las herramientas comerciales. Adicionalmente, el esfuerzo de openstreetmap.org de construcción de un mapa base comunitario del mundo, de acceso libre y con herramientas tipo open de desarrollo. Estas iniciativas potencian proyectos que antes no se contemplaban viables por los altos costos que demandaban [19].

Los GPS incorporados en Smartphone son herramientas que posibilitan múltiples tareas. Esto evidencia el gran potencial para la construcción de mapas colaborativos. Comercialmente, la empresa $W A Z E$, que recientemente fue adquirida por google, logra poner a su disposición una gran cantidad sensores GPS esparcidos por el mundo [20].

Un estudio realizado en 2014 sobre las tendencias de la información geográfica voluntaria para la investigación, muestra algunos datos estadísticos de proyectos colaborativos. En la Tabla 1 se detalla que solo WAZE y OpenStreetMap tienen cobertura mundial, por lo cual se analizan con mayor detalle en capítulos posteriores [16].

Entre los proyectos colaborativos descritos en la Tabla 1, destacan a nivel mundial OpenStreetMap y Waze. Estos cuentan con cobertura mundial que proporciona a los ciudadanos compartir datos e informarse. A continuación, se describen estos dos proyectos. 
Tabla 1

Comparación de Proyectos Colaborativos. Adaptado de [16].

\begin{tabular}{llllll}
\hline \hline Atributo & $\begin{array}{l}\text { Mapmaker } \\
(\text { Google) }\end{array}$ & $\begin{array}{l}\text { HereMap } \\
\text { Nokia }\end{array}$ & $\begin{array}{l}\text { MapShare } \\
\text { Tom Tom }\end{array}$ & $\begin{array}{l}\text { Waze } \\
\text { Google }\end{array}$ & $\begin{array}{l}\text { Open } \\
\text { Street Map }\end{array}$ \\
\hline Inicio & 2008 & 2012 & 2007 & 2008 & 2004 \\
\# Usuarios Registrados (millones) & N/A & N/A & 60 & 45 & 1.23 \\
Colaboradores Activos - mes (2013) & 40.000 & N/A & N/A & $12-13$ Mil- & 20.000 \\
& & & & lones & \\
Cobertura(\# Paises 2013) & $>220$ & $>120$ & $>90$ & WORLD & WORLD \\
Licencia & Propietaria & Propietaria & Propietaria & Propietaria & ODbL \\
¿Permite descargar datos? & NO & NO & NO & NO & SI \\
\hline \hline
\end{tabular}

\subsection{Mapas base de tipo colaborativo: WAZE}

Esta herramienta es una aplicación de tráfico y navegación. Los ciudadanos proporcionan la información del tráfico de las vías y pone a disposición de la comunidad para la toma de decisiones de rutas. Adicionalmente, habilita una herramienta web colaborativa para la edición del mapa base.

En octubre de 2014, WAZE lanzó el programa ciudadanos conectados, el cual está dirigido a las entidades gubernamentales y organismos sin ánimo de lucro, para liberar el uso de los datos que los ciudadanos aportan en WAZE, a través de un SDK (Software Developer Kit). Sin duda, es un avance importante, porque a partir de ese SDK se podrían estimar con cierto grado de precisión aforos de las vías y acceder a los datos asociados a las vías para la elaboración de mapas estratégicos de ruido [20].

\subsection{Mapas base colaborativo OPENSTREETMAP}

El proyecto OpenStreetMap (OSM) se inicia en 2004 con bases de datos y servicios web principales alojadas en una serie de servidores de la University College de Londres. El objetivo del proyecto es la construcción de una base de datos de libre acceso con información geográfica, que puede ser utilizado para propósitos de cartografía, navegación u otras aplicaciones. Actualmente, OSM cuenta con millones de colaboradores, los cuales comparten más de 5.000 millones de puntos GPS, 3.500 millones de nodos y 360 millones de vías, entre otros elementos geográficos [16].

La gran ventaja para proyectos colaborativos que tiene OSM es que permite adición de atributos, los cuales quedan almacenados como atributos asociados al objeto geográfico, al cual luego se puede acceder programáticamente. Lo anterior, permite adicionar las variables necesarias y no incluidas en el modelo de datos estándar de OSM, para poder modelar los mapas de ruido estratégicos objeto del presente artículo.

\section{Datos espaciales para la simulación de mapas de ruido estratégicos}

Un mapa estratégico de ruido se construye a partir de datos geográficos asociados al fenómeno. Esta información está definida para modelar las fuentes de ruido, el medio de propagación y receptores. Generalmente, estos datos son cartografía digital, ortofotografías en color, modelos digitales de terreno, carreteras, ferrocarriles, focos industriales u otros focos puntuales, edificaciones, población y usos del suelo [10]. Para la construcción de mapas estratégicos de ruido, la WG-AEN recomienda que para las fuentes de ruido ambiental tipo tráfico rodado se debe contar con la información que se detalla en la Tabla 2.

Los editores web existentes para la estructuración de la información de OSM pueden ser usados para el ingreso y estructuración de las variables básicas requeridas para la simulación de mapas de ruido estratégicos. La mayoría de los atributos requeridos están incluidos dentro del modelo estándar de OSM. Este proyecto por su cobertura global y creciente número de usuarios permite acceder a información actualizada cada vez que sea requerido. A continuación, se presentan estrategias colaborativas, para la adquisición de las variables que faltan en el modelo estándar de OSM.

\subsection{Fuentes de ruido}

Los atributos para los ejes viales, semáforos, estaciones de transporte masivo, rutas de transporte masivos están incluidos dentro del modelo estándar de OSM, con excepción de los datos de aforo, velocidad y los focos de ruido predominantes, por lo que solo es necesario concentrar los esfuerzos en estrategias para la obtención de esos datos [21, 22]. De manera especial se establecen las estrategias para realizar mapas de ruido de tráfico rodado.

Los datos de flujo de tráfico vehicular deben separarse en datos día y noche, además es importante separar los datos obtenidos en días de semana y los obtenidos en fines de semana. La caracterización vehicular contempla la diferenciación de vehículos (livianos, pesados y motos) en las diferentes 


\section{Tabla 2}

Información para la simulación de mapas estratégicos de ruido.

\begin{tabular}{|c|c|c|}
\hline Relacionada con Focos de Ruido & Nombres) & Atributos \\
\hline \multirow[t]{16}{*}{ Relacionada con Focos de Ruido } & Ejes Viales & Nombre \\
\hline & & Sentido \\
\hline & & Tipo \\
\hline & & Superficie \\
\hline & & Jerarquía \\
\hline & & Velocidad \\
\hline & & Aforo (día, noche) \\
\hline & & \# Carriles \\
\hline & & Ancho Carril \\
\hline & Rutas y estaciones de & Sentido \\
\hline & Transporte Masivo & Tipo Superficie \\
\hline & & Velocidad \\
\hline & & Aforo (día, noche) \\
\hline & & \# Carriles \\
\hline & & Ancho Carril \\
\hline & $\begin{array}{l}\text { Otros focos } \\
\text { predominantes }\end{array}$ & $\begin{array}{l}\text { Nivel de Potencia } \\
\text { Acústica }\end{array}$ \\
\hline \multirow[t]{7}{*}{ Relacionada con Focos de Ruido } & Curvas de nivel & $\begin{array}{l}\text { Altura sobre } \\
\text { el nivel del mar }\end{array}$ \\
\hline & Edificaciones & Tipo edificación \\
\hline & & Descripción \\
\hline & & Población \\
\hline & & Altura \\
\hline & & Numero pisos \\
\hline & Usos del suelo & Tipo \\
\hline \multirow[t]{2}{*}{ Relacionada con los receptores } & Limites & Nobre \\
\hline & $\begin{array}{l}\text { Delimitación área de es- } \\
\text { tudio }\end{array}$ & Área \\
\hline
\end{tabular}

jornadas del día, diurno y nocturno [2, 23]. En guías del país Vasco se recomienda que la campaña de recolección de aforos se realice por el tiempo suficiente para caracterizar adecuadamente el aforo vehicular [10]. Las variables relacionadas con la caracterización de la fuente tienen el mayor impacto en la incertidumbre de la simulación [4, 24].

Por ello, se propone una estrategia de adquisición de datos colaborativa basado en que las ciudades cada día incrementan el número de cámaras de seguridad y de vigilancia de tráfico, públicas o privadas. Además, los ciudadanos pueden aportar videos cortos, tomados desde sus teléfonos inteligentes que pueden programáticamente ser asociados a los segmentos viales. Estos videos analizados visualmente o por medio de herramientas sofisticadas de analítica de video, pueden aportar los datos de aforo necesarios para la simulación de mapas estratégicos de ruido [25].

Cada cámara que se encuentra en un segmento vial debe ser pensada para cumplir múltiples propósitos, los cuales van más allá de la simple inspección visual de lo que está pasando en la vía, para convertirse en una entrada de datos que proveen información relevante en múltiples proyectos que van desde el análisis estratégico de seguridad, movilidad y ruido

Algunos de los proyectos en esta área, tratan de solucionar tres problemas principales: clasificación, conteo y dirección. Trabajos como el de Maldonado, donde se construye un sistema automático de detección vehicular, soluciona los dos primeros problemas como aportes iniciales a la temática [26]. Este trabajo se centra en realizar un análisis posterior a la captura de video en la plataforma MatLab.

Actualmente en la web, se comparten algoritmos para la caracterización vehicular. Proyectos disponibles libremente en la web permiten integrar diferentes dispositivos para este fin. Es tal el caso que, herramientas como cámaras web, ordenadores de placa reducida como los Raspberry $\mathrm{Pi}$, y software como Simulink, permitan caracterizar el flujo vehicular en tiempo real y reportando los datos de manera inalámbrica [27]. Este tipo de proyectos, aunque reposan en la web de manera gratuita, falta que se desarrollen con programas de código abierto. 
De esta manera, se deben buscar alternativas para integrar la recolección de datos de flujo vehicular con los demás datos para realizar mapas de ruido.

Ejercicios como los descritos en [26], implementados en OpenCV (Open Source Computer Vision por autores como Andrews Sobra, proponen un método para la clasificación vehicular basado en propiedades de los vehículos a través de video. El uso de las redes neuronales artificiales proporciona gran exactitud en los resultados [28]. Este ejercicio utiliza herramientas de código libre, lo que facilita su inclusión dentro de las herramientas que posibilitan el acceso a los datos requeridos para la simulación de mapas de ruido.

\subsection{Propagación del ruido}

Para modelar la propagación del ruido se requieren dos variables básicas, las cuales son las curvas de nivel y las edificaciones. Para su adquisición se proponen las siguientes estrategias colaborativas:

\subsubsection{Curvas de nivel}

Muchos proyectos OSM usan datos Shuttle Radar Topography Mission (SRTM), misión de la NASA que inicio desde el año 2000, para obtener datos de elevación del mundo), para proveer la información de curvas de nivel, un ejemplo es OpenCycleMap, el cual muestra curvas de nivel y relieve sombreado obtenido del STRM. Srtm20sm es una herramienta libre que utiliza SRTM para generar curvas de nivel de un terreno seleccionado [29].

\subsubsection{Edificaciones}

Actualmente, existen varias aplicaciones disponibles que proporcionan información de OSM en 3D, pero la más avanzada que contiene una amplitud de escenarios gráficos y especificaciones Open Geospatial Consortium(OGC) y servicios Web Map Service (WMS) o Web 3D Service (W3DS) es OSM-3D [30]. Con estas herramientas se puede modelar y visualizar dentro de OSM edificaciones en 3D. Sin embargo, con la simple edición de niveles de las edificaciones dentro del modelo de datos estándar de OSM se puede obtener una aproximación simple de la altura de las edificaciones, multiplicando el número de niveles por una altura promedio por nivel. El número de niveles de piso es fácilmente observable desde aplicaciones como Street View de Google o pueden ser adquiridas de mapas prediales catastrales del área de estudio. El número de viviendas por edificación y habitantes puede ser determinada de datos públicos. Los entes gubernamentales disponen en sus sistemas de información datos de densidad de vivienda y población por municipio. En el caso de Colombia el Departamento Nacional de Estadística dispone de estimaciones y proyecciones de la población [31].

\section{Caso de aplicación}

Con el propósito de acercar la propuesta metodológica a una adquisición de datos de tipo colaborativo, se define un área de estudio contigua a la sede San Benito de la Universidad San Buenaventura Medellín, tal y como se aprecia en la Figura 1.
Figura 1. Zona de estudio visualizada en OSM.

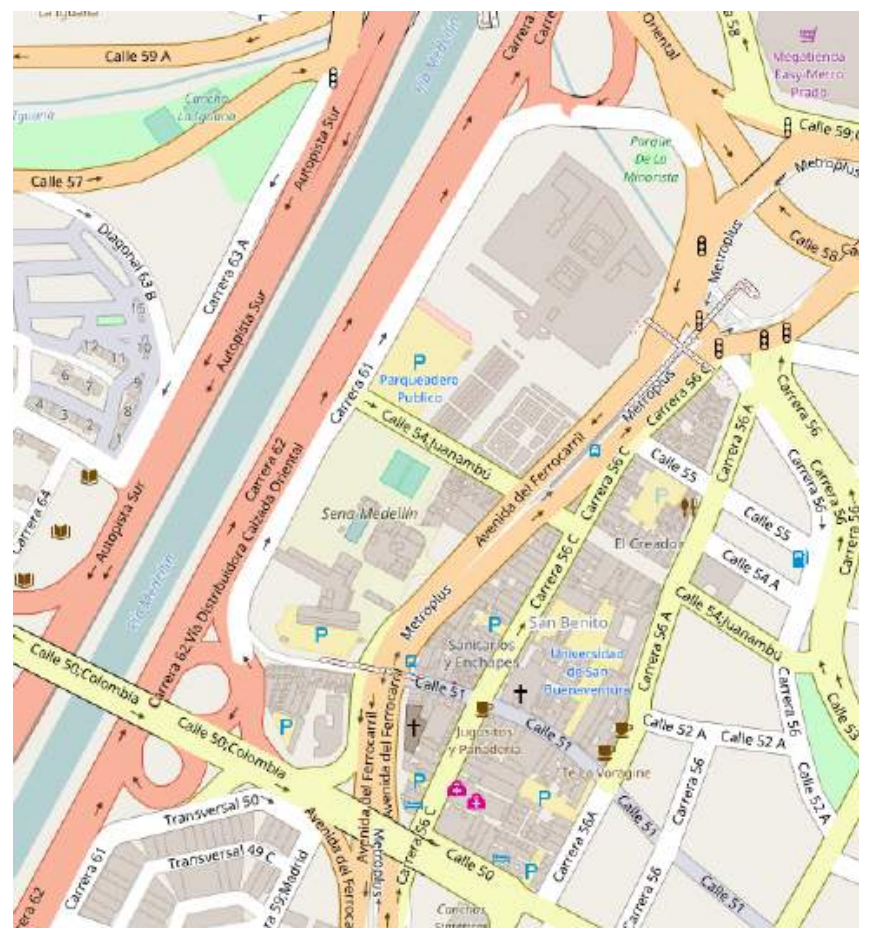

A través del software JOSM, se realiza una edición offline para cargar masivamente las ediciones a OSM. Se crearon los polígonos de las edificaciones y se adicionaron los atributos necesarios para la modelación del mapa de ruido. Las vías también son editadas, agregando los atributos requeridos como se aprecia en la Figura 2.

Con la adición de estos elementos al OSM, se realiza una visualización del modelo 3D. Usando el complemento web de OSM denominado "osmbuildings" es posible visualizar el modelo de acuerdo a las capas y atributos editados en el software JOMS y cargados en OSM (ver Figura 3).

Mediante el uso de QGIS, se importan los datos espaciales al sistema de información geográfica. Esto tiene como objetivo verificar la distribución de los atributos y conjugación con datos dispuestos en OSM que son necesarios en el software de predicción acústica. En Figura 4, se puede apreciar los datos extraídos del OSM y dispuestos en QGIS.

Con los datos en el Sistema de Información QGIS, se realiza la edición del área de estudio para definir los atributos necesarios tanto en las vías como en las edificaciones. Se toma una sola vía (Carrera $56 \mathrm{C}$, que tiene el flujo vehicular en sentido surnorte), en donde el aforo vehicular se determina por medio de análisis de video tomado desde una edificación aledaña (ver Figura 5).

A partir del análisis del video se obtiene el número de vehículos livianos (Carros y motos) y pesados (camiones y buses) que transitaban por la vía en un periodo de 2 minutos, a través de algoritmo de conteo en OpenCV y manualmente. Con estos datos se determina el número de vehículos para una jornada diurna 14 horas. En la Tabla 3 se presentan los resultados del 
Figura 2. Edición de edificaciones en JOSM.

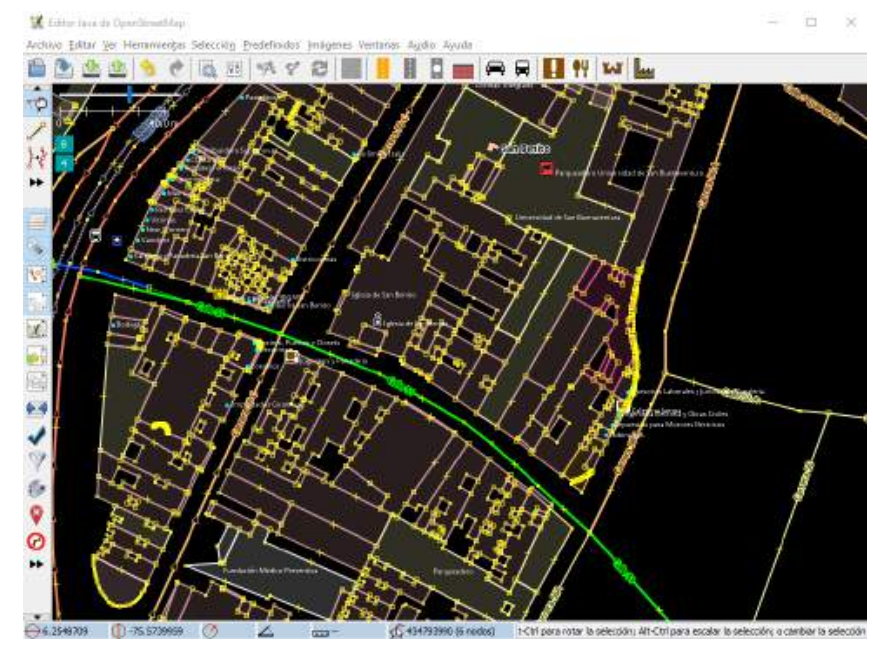

Figura 3. Edición de edificaciones en JOSM.

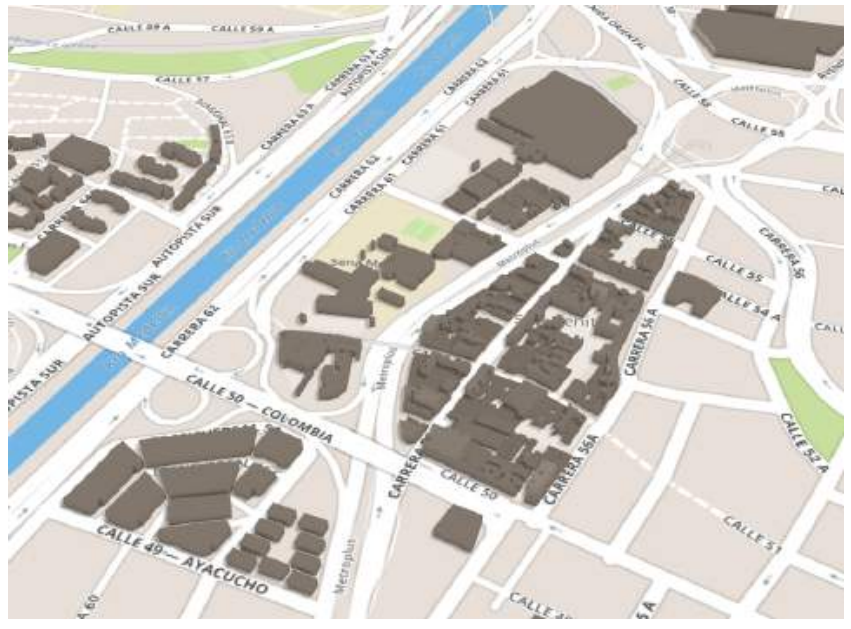

conteo de vehículos.

Para las edificaciones se extrae el dato de número de pisos de uno de los atributos importados denominado "other tags", por medio del uso del complemento FieldPyculator de QGIS. Con esta herramienta se crea un nuevo atributo que contuviera el número de pisos de cada polígono que representa una edificación. Así mismo, se define la altura de cada edificio según el número de pisos y altura promedio de cada uno de 2.5 metros. Adicionalmente, se crea un polígono que representa el área de cálculo, y otro para las curvas de nivel. Los datos son exportados en un archivo tipo shape, para ser dispuestos en el software SoundPLANS.

En el Software SoundPLANS se crean ficheros para cada una de las capas extraídas en QGIS. Cada atributo es relacionado dentro del software de simulación, además se ajustan los que no fueron extraídos del OSM, como el aforo vehicular (tomado del análisis de video), característica de la vía y del terreno. En la Figura 5 se presenta la visualización de la situación creada a partir de datos colaborativos para la deter-
Figura 4. Visualización vías en QGIS del área de estudio.

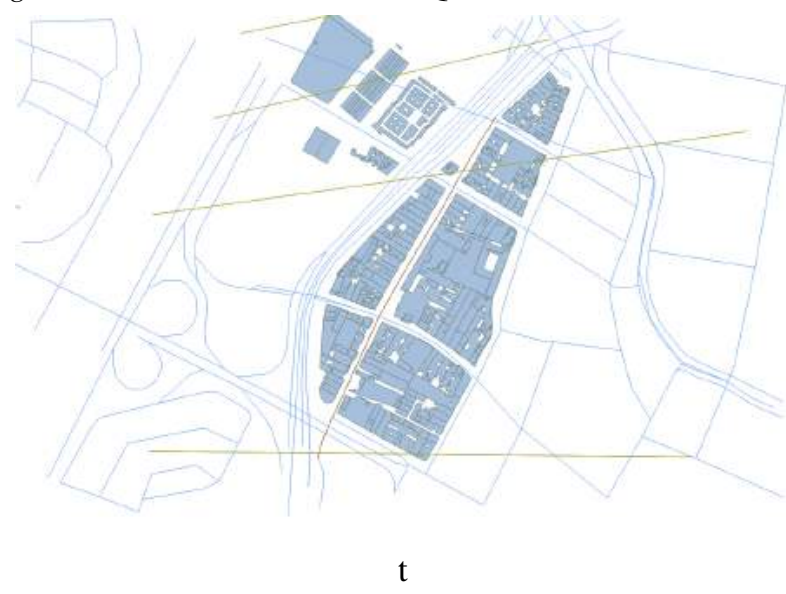

Figura 5. Fotograma del video adquirido con un teléfono celular de la Carrera $56 \mathrm{C}$.

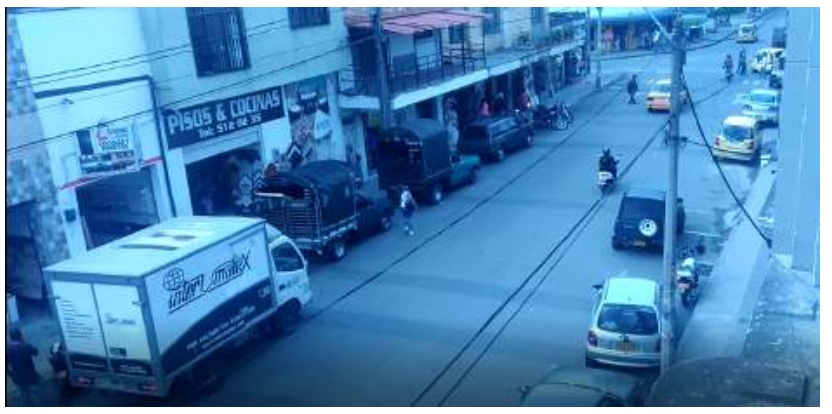

minación de los niveles de ruido a 4 metros de altura, producto del tráfico vehicular.

Se establece para el cálculo para el ruido de carretera el método alemán RLS-90. La malla de cálculo es definida como 5 metros y la altura del receptor es definida a 4 metros sobre el terreno. En la Figura 6 se presenta el resultado de la simulación realizada en Soundplan ${ }^{\circledR}$ con datos colaborativos para el periodo diurno en la vía que cruza de sur a norte la zona de estudio.

\section{Conclusiones}

De la revisión y análisis de los diferentes proyectos colaborativos disponibles a nivel mundial, se identifica la posibilidad de adquirir los datos para la construcción de un modelo base que permita la modelación y simulación de mapas de ruido. Estos proyectos son la base para la homogeneización y normalización de datos para la representación dinámica de los mapas.

Los instrumentos colaborativos significativos para abordar la adquisición de datos para la simulación de mapas de ruido son OpenStreetMap, los datos de Shuttle Radar Topography Mission y Open Computer Visión. Las integraciones de estas iniciativas deberán proporcionar los datos requeridos, que después de su adecuación permiten incluir modelos de predicción sonora para la estimación de niveles de ruido. 
Tabla 3

Estimación de aforo vehicular de la carrera 56 C.

\begin{tabular}{llll}
\hline \hline Periodo & \multicolumn{2}{c}{ Vehículos Livianos } & $\begin{array}{l}\text { Vehículos } \\
\text { pesados }\end{array}$ \\
\hline 2 minutos & 12 & 10 & 2 \\
1 hora & 360 & 300 & 60 \\
14 horas & 5040 & 4200 & 840 \\
TOTAL & & $\mathbf{9 2 4 0}$ & $\mathbf{8 4 0}$ \\
\hline \hline
\end{tabular}

Figura 6. Visualización de la situación creada para la simulación del mapa de ruido en SoundPLAN ${ }^{\circledR}$ con los datos extraído de los sistemas colaborativos.

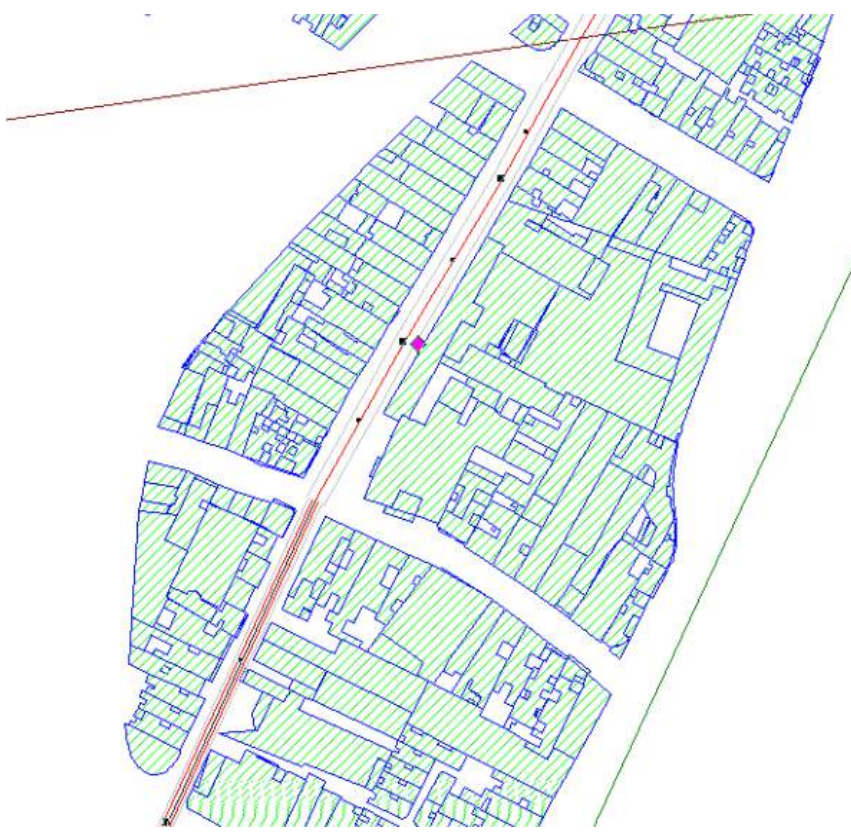

La discusión presentada da pie al uso de herramientas de código libre para fines como la construcción de mapas estratégicos de ruido. La información recolectada debe obedecer las diferentes guías propuestas por diferentes grupos de trabajo a nivel mundial. Esto tiene como fin de que los datos sean normalizados para la correcta predicción del ruido ambiental.

\section{References}

[1] A. E. González-Bach and R. Gerardo, "Contaminación física en ambiente urbano: el ruido," Dep. Ing. Ambient IMFIA Fac. Ing. la Univ. la República, 2016.

[2] P. H. T. Zannin and D. Q. de Sant'Ana, "Noise mapping at different stages of a freeway redevelopment project a case study in brazil," Applied Acoustics, vol. 72, no. 8, pp. $479-486,2011$.
Figura 7. Resultado mapa de Ruido de tráfico rodado realizado con datos colaborativos en el software Soundplan.

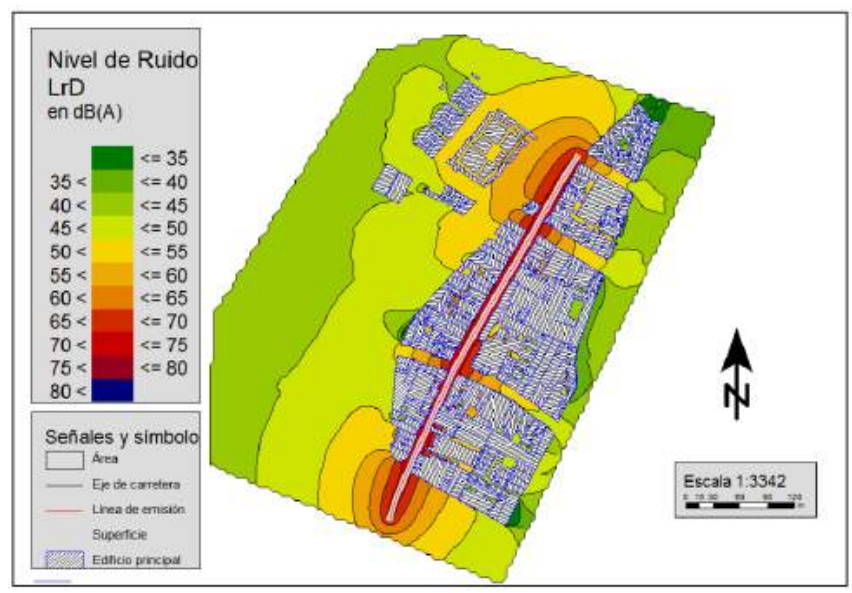

[3] O. M. de la Salud, Guías para el ruido urbano. OMS, 1999.

[4] E. C. W. G. A. of Exposure to Noise (WG-AEN), "Good practice guide for strategic noise mapping and the production of associated data on noise exposure - position paper," 2006.

[5] P. Europeo, "Directiva 2002/49/ce del parlamento europeo y del consejo de 25 de junio de 2002 sobre evaluación y gestión del ruido ambiental," 2002.

[6] D. M. Murillo-Gómez, "Resolución espacial en la elaboración de mapas de ruido por interpolación," Ing. Ing. USBMed, vol. 1, no. 1, pp. 56-62, 2017.

[7] AMVA and C. PS-AAC, Actualización de los mapas de ruido de la zona urbana de los municipios de Medellín, Bello e Itagüi. AMVA, 2015.

[8] C. R. Alves-Monteiro, "Ruido ambiental y paisaje sonoro. sinergias en el medio urbano," Master's thesis, Universidad de Valladolid, Septiembre 2013.

[9] F. S. Echazarreta, "Estrategia de elaboración de un mapa de ruido," 2008.

[10] Departamento de Ordenación del Territorio y Medio Ambiente, "Guía metodológica para la realización de mapas de riudo," 2005.

[11] A. M. Jiménez and P. S. Martínez, "El ruido ambiental urbano en madrid. caracterización y evaluación cuantitativa de la población potencialmente afectable," Boletín de la Asoc. Geógrafos Españoles, pp. 153-180, 2005.

[12] C. de Abreu Freire and M. Painho, "Development of a mobile mapping solution for spatial data collection using open-source technologies," Procedia Technology, vol. 16, pp. $481-490,2014$.

[13] J. P. G. Barrón, "Crowdsourcing de información geográfica: proyectos open source y desarrollo de una 
plataforma móvil para mantenimiento de infraestructura urbana," Master's thesis, E.T.S.I. en Topografía, Geodesia y Cartografía (UPM), 2014.

${ }^{[14]}$ D. Sui, S. Elwood, and M. Goodchild, Crowdsourcing Geographic Knowledge. Springer Netherlands, 1 ed., 2013.

[15] M. E. Scarpa, "Google maps cumple 11 años revolucionando la cartografía mundial," La Tercera, 2016.

[16] P. Neis and D. Zielstra, "Recent developments and future trends in volunteered geographic information research: The case of openstreetmap," Future Internet, vol. 6, p. 76-106, Jan 2014.

[17] Esri, "El sistema arcgis," 2016.

${ }^{[18]}$ I. Corporation, "Acerca de intergraph."

[19] S. Steiniger and A. J. Hunter, "The 2012 free and open source gis software map - a guide to facilitate research, development, and adoption," Computers, Environment and Urban Systems, vol. 39, pp. 136 - 150, 2013.

[20] Waze, "Conectando ciudadanos y gobiernos a través de datos," 2016.

[21] J. H. Ko, S. I. Chang, and B. C. Lee, "Noise impact assessment by utilizing noise map and gis: A case study in the city of chungju, republic of korea," Applied Acoustics, vol. 72, no. 8, pp. 544 - 550, 2011.

[22] P. Pamanikabud and M. Tansatcha, " $3 \mathrm{~d}$ analysis and investigation of traffic noise impact from a new motorway on building and surrounding area," Applied Acoustics, vol. 71, no. 12, pp. 1185 - 1193, 2010.

[23] J. Rendón, J. R. Gómez, A. F. Monsalve, and D. M. Murillo, "Índices de ruido urbano en el día sin carro en la ciudad de medellín," Ing. USBMed, vol. 1, no. 1, 2010.

[24] D. M. Murillo-Gómez, J. C. Gil-Carvajal, V. ZapataRodríguez, and J. J. Téllez-García, "Assessment of the rls 90 calculation method for predicting road traffic noise in colombian conditions," Rev. Fac. Ing, vol. 1, no. 75, pp. 175-188, 2015.

${ }^{[25]}$ M. J. M. Chan, Sistema automático de conteo y clasificación de flujo vehicular basado en secuencias de video $y$ redes neuronales artificiales. 2006.

[26] M. Maldonado, Universidad Autónoma de Nuevo León División de Estudios de Posgrado, ch. Sistema Automático de Conteo y Clasificación de Flujo vehicular Basado en Secuancias de Video y Redes Neuronales Artificiales. 2006.

[27] E. Wetjen, "Counting cars and analyzing traffic with a raspberry pi, a webcam and thingspeak," 2015.

[28] A. Sobral, L. Oliveira, L. Schnitman, and F. Souza, "Highway traffic congestion classification using holistic properties," Int. Conf. Signal Process. Pattern Recognit. Appl, pp. $458-465,2015$.
[29] M. Goetz and A. Zipf, "Openstreetmap in 3d-detailed insights on the current situation in germany," AGILE, pp. 24-27, 2012.

${ }^{[30]}$ M. Over, A. Schilling, S. Neubauer, and A. Zipf, "Generating web-based $3 \mathrm{~d}$ city models from openstreetmap: The current situation in germany-," Comput. Environ. Urban Syst, vol. 34, no. 6, pp. 496-507, 2010.

[31] (Departamento Administrativo Nacional de Estadística (DANE), "Estimaciones y proyecciones de población," 2016. 